\title{
Classifying an Object using Class Differentiators
}

\author{
${ }^{1}$ Seunghyun Im, ${ }^{2}$ Li-Shiang Tsay \\ ${ }^{1}$ Computer Science Department, University of Pittsburgh at Johnstown, USA; \\ ${ }^{2}$ Department of Computer Systems Technology, North Carolina A\&T University, USA; \\ sim@pitt.edu; Itsay@ncat.edu
}

\begin{abstract}
This paper discusses a supervised classification method. The method classifies an object using class differentiators. The class differentiators are the smallest set of values in a class that effectively distinguish one class from the others. The class membership is determined by the degree of homology between the test object and the class differentiators. Unlike many rule based classifiers, the algorithm proposed in this paper does not require input parameters and always produces the same results from the same data set. The algorithm is designed to work with categorical data, and is particularly useful when the quantification of the data is infeasible. We present an experimental result to show the validity of the algorithm.
\end{abstract}

Keywords: Classification algorithm, Supervised Classifier, Categorical Data, Reduct, Rough Set.

\section{Introduction}

We present a supervised classification method for categorical data. The classification problem in this paper focuses on the prediction of the class of a test object. We assume that the training data set has condition and decision attributes. The class labels of the training data set are determined by the discrete values in the decision attribute. The class label of the test object is uncertain. We use the training data to predict the unknown class label of the test object. The prediction can be performed by measuring the similarity (or distance) between objects. The data type has great influence on the similarity measure. For categorical data, the method can be divided into two types (1) discrete similarity measure and (2) approximated similarity measure. In general, the similarity between two attribute values is either 1 or 0 in discrete similarity measure; 1 for the same symbols and 0 for the different symbols between two objects. Therefore, the amount and frequency of the overlap between the training data and the newly observed object determine how much the object is close to a class. The classifiers based on the classical Naive Bayes [3] or Classification Rule [4] fall into this category. On the other hand, the methods derived from the approximated similarity measure convert the categorical data to numeric data in order to measure the degree of similarities between non-identical symbols. (e.g. the distance between red and blue is 0.7). Many algorithms have been proposed to address the conversion problem [5]. Although they are fundamentally useful, the quantification of categorical data is still challenging due to the fact that distances are often too arbitrary or it requires an intensive phase of data preprocessing, such as discretization of data [6] or construction of ontology [7][8]. The quantification is even more difficult when data are sanitized for privacy and regulatory requirements [9][10] because we do not know the meaning of the symbols. This is the case with many dataset from medical area. The algorithm presented in this 
paper falls into the discrete similarity measure category. We developed this algorithm for the classification task that quantification is not possible.

The rule based classifier is one of the most widely studied supervised classifier for categorical data. A number of algorithms have been proposed [11]. These algorithms generate if-then style rules, and use the antecedent to measure the similarity and the consequent to predict the class label. Despite being very useful in many applications, these methods require quality threshold values, e.g. minimum support and confidence value for rule extraction. The determination of the optimal threshold value requires a deep understanding of the data and often need help from the domain expert. Another potential problem is that the result of the classification varies depending on the input parameters because the changes in these parameters result in different set of rules. The proposed algorithm does not require input parameters. This is an advantage for the classification task that requires consistent result or for non-technical users who are not familiar with the notion of the threshold value. Naive Bayes classifier is another well-known classification algorithm. It is simple and easy to use. We believe our method is as simple as the classical Naive Bayes classifier, yet it does not need any type of pseudo-count to solve the zero frequency problem.

The proposed algorithm is inspired by the concept of object reduct [2]. An object reduct is the minimal set of values of an object which differentiates the object from other objects. We extended the concept to detect patterns characterizing each class, and use them to find the most promising class label for a newly observed object. We present our method in Chapter 2, an experimental result in Chapter 3, and the conclusion in Chapter 4.

Table 1: Example of Information System.

\begin{tabular}{l|llll}
\hline & $B$ & $C$ & $E$ & $D$ \\
\hline$x_{1}$ & $b_{1}$ & $c_{1}$ & $e_{1}$ & $d_{1}$ \\
$x_{2}$ & $b_{2}$ & $c_{1}$ & $e_{2}$ & $d_{2}$ \\
$x_{3}$ & $b_{2}$ & $c_{3}$ & & $d_{2}$ \\
$x_{4}$ & $b_{1}$ & $c_{1}$ & $e_{1}$ & $d_{2}$ \\
\hline
\end{tabular}

\section{Algorithm}

\subsection{Basic Notations}

We will use the following notations for dataset. By an information system [1] we mean a triple $S=(X, A, V)$, where

$X=\left\{x_{1}, x_{2}, \ldots, x_{i}\right\}$ is a finite set of objects,

$A=\left\{a_{1}, a_{2}, \ldots, a_{j}\right\}$ is a finite set of attributes

$V=\left\{v_{1}, v_{2}, \ldots, v_{k}\right\}$ is a finite set of attribute values.

The information system in Table 1 has 4 objects $X=\left\{x_{1}, x_{2}, x_{3}, x_{4}\right\}$ and 4 attributes $A=\{B, C, E, D\}$. The attributes are partitioned into two types: condition and decision. The condition attributes are $B, C$, and $E$, and the decision attribute is $D$. They are written as,

$A_{C}=\{B, C, E\} \quad$ condition attribute

$A_{D}=\{D\} \quad$ decision attribute 
Seunghyun Im \& Li-Shiang Tsay; Classifying an Object using Class Differentiators, Transactions on Machine Learning and Artificial Intelligence, Volume 2 No 5 Oct (2014); pp: 64-71

The following notations are used for attribute values.

$B\left(x_{1}\right)=b_{1} \quad b_{1}$ is the value of $B$ in $x_{1}$

$V_{D}=\left\{d_{1}, d_{2}\right\} \quad$ attribute values for attribute $D$

We assume that the classification is guided by the decision values. Therefore, a class is a set of objects in $S$ with the same decision value, which is written as,

$X_{d}=\left\{x_{k} \in X ; D\left(x_{k}\right)=d\right\} \quad$ Class defined by decision value $d$

In Table $1, X_{d 1}=\left\{x_{1}\right\}$ and $X_{d 2}=\left\{x_{2}, x_{3}, x_{4}\right\}$. We use an information system that has two decision values $\left(d_{1}\right.$ and $d_{2}$ ) for simplicity of presentation, but the algorithm directly carries over to the general case where $\left|V_{D}\right| \geq 2$.

\subsection{Method Description}

\subsubsection{Differentiators}

The differentiator used in this paper is a derivation of reduct [2] in rough set theory [1]. An object-object differentiator, denoted as $\Delta_{o o}\left(x^{\prime}, x^{\prime \prime}\right)$, is the disjunction of the attribute values in object $x^{\prime}$ that distinguishes $x^{\prime}$ from the other object $x^{\prime \prime}$. For example, in Table 1 , we can easily distinguish $x_{1}$ from $x_{2}$ with $b_{1}$ of $B$. i.e., a person wearing a red shirt $\left(b_{1}\right)$ from another person wearing a blue shirt $\left(b_{2}\right)$. We do not need other attribute values to distinguish $x_{1}$ from $x_{2} . \Delta_{o o}\left(x_{1}, x_{2}\right)$ is $b_{1}$ or $e_{1}$, and it is written as $\left(b_{1} \vee e_{1}\right)$. Although we have two attribute values, one is enough to discern $x_{1}$. An object may contain null values. We use two methods for handling the null value: (1) if an attribute value of $x^{\prime \prime}$ is null the corresponding attribute value in $x^{\prime}$ becomes an element of $\Delta_{o o}\left(x^{\prime}, x^{\prime \prime}\right)$ because the null value in $x^{\prime \prime}$ is different from the value in $x^{\prime}$. (2) if an attribute value in $x^{\prime}$ is null we do not add the null to $\Delta_{o o}\left(x^{\prime}, x^{\prime \prime}\right)$ because a null is not a specific value that can distinguish $x^{\prime}$ from other objects.

An object-class differentiator, denoted as $\Delta_{o c}\left(x^{\prime}, X_{d^{\prime \prime}}\right)$, is the shortest terms that distinguish $x^{\prime}$ from $X_{d^{\prime \prime}}$ (e.g. $x_{1}$ and $\left.X_{d 2}=\left\{x_{2}, x_{3}, x_{4}\right\}\right)$. The shortest terms are acquired by finding the prime implicants [12] of all objectobject differentiators $\left(\Delta_{0}\right)$ between $x^{\prime}$ and $\left\{x \in X_{d^{\prime \prime}}\right\}$. A prime implicant is a product term that cannot be subsumed by any other product term. To obtain the prime implicant set, we put all $\Delta_{o o s}$ into conjunctive normal form (CNF) and transform it to disjunctive normal forms (DNF). $\Delta_{0 o}=\emptyset$ when two objects are identical. In this case, we do not include the empty set as a conjunct of the CNF. The object-class differentiator $(\Delta o c)$ consisting of at least one empty $\Delta_{o o}$ is called approximated object-class differentiator. Otherwise, it becomes a precise object-class differentiator. When $x^{\prime}$ is compared with all other objects in $\left\{X-x^{\prime}\right\}$ it is simply written as $\Delta_{o c}\left(x^{\prime}\right)$. Intuitively, $\Delta_{o d}\left(x^{\prime}\right)$ is the attribute values in $x^{\prime}$ that can most effectively (not necessarily precisely) differentiate $x^{\prime}$ from the other objects in $S$.

A class-class differentiator, denoted as $\Delta_{c c}\left(X_{d^{\prime}}, X_{d^{\prime \prime}}\right)$, is the disjunction of $\Delta_{O c} s$. It is the set of unique terms in $X_{d^{\prime}}$ in relation to $X_{d^{\prime \prime}}$. A $\Delta_{c c}\left(X_{d^{\prime}}, X_{d^{\prime \prime}}\right)$ is categorized as an approximated class-class differentiator if it has one or more approximated $\Delta o c$. Otherwise, it becomes a precise class-class differentiator. Some terms may appear more than once in a $\Delta_{c c}$. The frequency of a term $t$ is defined as the number of times $t$ is a subset of $x$ in $X_{d}$ 


$$
f(t)=\sum_{i=1}^{n} \begin{cases}1, & t \subseteq x_{i}: x_{i} \in X_{d} \\ 0, & \text { otherwise }\end{cases}
$$

When $X_{d^{\prime}}$ of $\Delta_{c c}\left(X_{d^{\prime}}, X_{d^{\prime \prime}}\right)$ is $X-X_{d^{\prime \prime}}$ (e.g. there are only 2 class labels), the class differentiator of $X_{d^{\prime}}$ is simply written as $\Delta_{C C}\left(X_{d^{\prime}}\right)$. Let $\left(t_{i}, f_{i}\right)$ be a term and its frequency. Then, $\Delta_{c C}\left(X_{d^{\prime}}\right)$ is,

$$
\Delta_{c}\left(X_{d^{\prime}}\right)=\left\{\left(t_{1}, f_{1}\right),\left(t_{2}, f_{2}\right), \ldots,\left(t_{n}, f_{n}\right)\right\}
$$

We use $\Delta_{c c}\left(X_{d}\right)$ to classify a test object.

\subsubsection{Classifying a test object}

Suppose that we want to classify the object $x_{\text {new }}$. The class label of $x_{\text {new }}$ is determined by its unknown decision value. We calculate the value by measuring how similar $x_{n e w}$ is to the terms in the class differentiators. For example, the decision value of $x_{n e w}$ is $d_{1}$ if $x_{\text {new }}$ is closer to the terms of $\Delta_{c c}\left(X_{d 1}\right)$ than those of $\Delta_{c c}\left(X_{d 2}\right)$. The idea is that $\Delta_{c c}\left(X_{d 1}\right)$ is the distinctive pattern of values that characterizes the class. If the same pattern is found in $x_{n e w}, x_{n e w}$ is most likely having the same characterestics of $\Delta_{c c}\left(X_{d 1}\right)$. Since the example problem has two class differentiators, we measure the similarity between the terms of $x_{n e w}$ and the terms of $\Delta_{c c}\left(X_{d^{\prime}}\right)$ and $\Delta_{c c}\left(X_{d^{\prime \prime}}\right)$ respectively. Then, we compare the degree of similarity to choose the right decision value. The degree of similarity, expressed as a weight, is calculated by the ratio between the sum of the frequencies of the terms in $\Delta_{c c}\left(X_{d}\right)$ and the number of the objects in $X_{d}$. Let $\omega^{x_{n e w}}(d)$ be the weight of the unknown decision value of $x_{n e w}$. Then,

$$
\omega^{x_{\text {new }}}(d)=\frac{\sum f\left(t_{i}\right): t_{i} \subseteq x_{\text {new }}, t_{i} \in \Delta_{C C}\left(X_{d}\right)}{\left|X_{d}\right|}
$$

Next, we compare the weights of all decision values. The object $x_{\text {new }}$ belongs to a class that has the highest $\omega^{x}$ new.

$$
x_{\text {new }} \in X_{d}: \omega^{x_{\text {new }}}(d)=\max \left(\omega^{x_{\text {new }}}\left(d_{i}\right)\right), i=1 \ldots\left|V_{D}\right|
$$

When two or more decision values have the equal weight, we randomly select one. We show an example in the next section.

\subsection{Sample Problem}

We will use the information system in Table 2. $A_{C}=\{B, C, E\} . A_{D}=\{D\}$. The dashed line indicates the division between two classes. $X_{d 1}=\left\{X_{1}, X_{2}, X_{3}, X_{4}\right\}$ and $X_{d 2}=\left\{X_{5}, X_{6}, X_{7}\right\} . x_{n e w}$ is the object to be classified.

Table 2: Information System S and an object Xnew

\begin{tabular}{l|lll|l}
\hline & $B$ & $C$ & $E$ & $D$ \\
\hline$x_{1}$ & $b_{2}$ & $c_{1}$ & $e_{1}$ & $d_{1}$ \\
$x_{2}$ & $b_{2}$ & $c_{3}$ & $e_{1}$ & $d_{1}$ \\
$x_{3}$ & $b_{1}$ & $c_{1}$ & & $d_{1}$ \\
$x_{4}$ & $b_{1}$ & $c_{3}$ & $e_{1}$ & $d_{1}$ \\
\hdashline$x_{5}$ & $b_{1}$ & $c_{1}$ & $e_{1}$ & $d_{2}$ \\
$x_{6}$ & $b_{1}$ & $c_{1}$ & $e_{1}$ & $d_{2}$ \\
$x_{7}$ & $b_{2}$ & $c_{2}$ & $e_{2}$ & $d_{2}$ \\
\hline$x_{\text {new }}$ & $b_{2}$ & $c_{3}$ & $e_{1}$ & \\
\hline
\end{tabular}


Seunghyun Im \& Li-Shiang Tsay; Classifying an Object using Class Differentiators, Transactions on Machine Learning and Artificial Intelligence, Volume 2 No 5 Oct (2014); pp: 64-71

We first build a discernibility matrix from Table 2 in order to obtain $\Delta_{c c}\left(X_{d 1}\right)$. Table 3 is the discernibility matrix that the elements in each cell are the object-object differentiators. For example, $b_{2}$ is the attribute value that discerns $x_{1}$ from $x_{5}$ in Table 2. Then, $b_{2}$ is placed in the cell between $x_{1}$ and $x_{5}$ in Table 3. Either $c_{1}$ or $e_{1}$ can distinguish $x_{1}$ from $x_{7}$ (or is denoted as $\vee$ sign), and they are placed between $x_{1}$ and $x_{7}$ in Table 3.

Table 3: Discernibility matrix for $\mathrm{X}_{\mathrm{d} 1}$

\begin{tabular}{|l|l|l|l|l|}
\hline & $x_{1}$ & $x_{2}$ & $x_{3}$ & $x_{4}$ \\
\hline$x_{5}$ & $b_{2}$ & $b_{2} \vee c_{3}$ & & $c_{3}$ \\
\hline$x_{6}$ & $b_{2}$ & $b_{2} \vee c_{3}$ & & $c_{3}$ \\
\hline$x_{7}$ & $c_{1} \vee e_{1}$ & $c_{3} \vee e_{1}$ & $b_{1} \vee c_{1}$ & $b_{1} \vee c_{3} \vee e_{1}$ \\
\hline
\end{tabular}

The conjunction of all $\Delta_{o o}\left(x_{1}\right) s$ is $\left(b_{2}\right) \wedge\left(b_{2}\right) \wedge\left(c_{1} \vee e_{1}\right)$. We transform it to a DNF to find $\Delta_{o c}\left(x_{1}\right)$. That is, $\Delta o c\left(x_{1}\right)=\left(b_{2}\right) \vee\left(b_{2}\right) \vee\left(c_{1} \wedge e_{1}\right)=\left(b_{2} \wedge e_{1}\right) \vee\left(b_{2} \wedge c_{1}\right)$

We also compute $\Delta_{\mathrm{oc}}$ for $x_{2}, x_{3}, x_{4}$.

$\Delta_{O C}\left(x_{2}\right)=\left(b_{2} \wedge c_{3}\right) \vee\left(b_{2} \wedge c_{3}\right) \vee\left(c_{3} \wedge e_{1}\right)=\left(c_{3}\right) \vee\left(b_{2} \wedge e_{1}\right)$

$\Delta o c\left(x_{3}\right)=\left(b_{1} \wedge c_{1}\right)$

$\Delta_{O C}\left(x_{4}\right)=\left(c_{3}\right) \wedge\left(c_{3}\right) \wedge\left(b_{1} \wedge c_{3} \wedge e_{1}\right)=\left(c_{3}\right)$

Next, we calculate the frequency of the terms in $\Delta_{o o s}$ to build $\Delta_{c c}\left(d_{1}\right)$. For instance, the frequency of $c_{3}$ is 2 because it is in $\Delta_{O C}\left(x_{2}\right)$ and $\Delta_{O C}\left(x_{4}\right)$. We can find $b_{2} \wedge e_{1}$ twice in $\Delta_{O C}\left(x_{1}\right)$ and $\Delta_{O C}\left(x_{2}\right)$. Its frequency is 2 . Thus, $\Delta_{c c}\left(d_{1}\right)$ is,

$\Delta_{c C}\left(d_{1}\right)=\left\{\left(c_{3}, 2\right),\left(b_{2} \wedge e_{1}, 2\right),\left(b_{2} \wedge c_{1}, 1\right),\left(b_{1}, 1\right),\left(c_{1}, 1\right)\right\}$

The class-class differentiator for $d_{2}, \Delta_{c}\left(d_{2}\right)$, can be obtained from the same matrix because there are only 2 decision values in $S$ and the discernibility matrix is symmetric. Using the same method we acquire,

$\Delta_{c c}\left(d_{2}\right)=\left\{\left(b_{1} \wedge c_{1} \wedge e_{1}, 2\right),\left(c_{2}, 1\right),\left(e_{1}, 1\right)\right\}$

Table 4 shows the terms, frequencies, and class labels of $\Delta_{c c}\left(d_{1}\right)$ and $\Delta_{c c}\left(d_{2}\right)$. We calculate the weight of the class label of $x_{n e w}$ using Table 4 . A term in Table 4 is counted to compute the weight if the term is a subset of $x_{n e w}$. We can find that term $\# 1=\left\{c_{3}\right\}$ and term $\# 2=\left\{b_{2}, e_{1}\right\}$ are the subsets of $\left\{b_{2}, c_{3}, e_{1}\right\}$. Their frequencies are 2 and 2 respectively. There are 4 objects in $X_{d 1}$. Therefore, the weights of $d_{1}$ for $x_{n e w}$ is,

$$
\omega^{x_{n e w}}\left(d_{1}\right)=\frac{2+2}{4}
$$

In the same way, term \#8 is a subset of $x_{n e w}$, and we use its frequency value 1 and the number of terms in $X_{d 2}$ to calculate the weight of $d_{2}$.

$$
\omega^{x_{n e w}}\left(d_{2}\right)=\frac{1}{3}=0.33
$$

We classify $x_{\text {new }}$ to $X_{d 1}$ because $\omega^{x_{\text {new }}}\left(d_{1}\right)>\omega^{x_{\text {new }}}\left(d_{2}\right)$. 
Table 4. Class differentiator $(\Delta \mathrm{CC})$ for $\mathrm{S}$

\begin{tabular}{|c|c|c|c|}
\hline term \# & term & frequency & class label \\
\hline 1 & $c_{3}$ & 2 & \multirow{2}{*}{${ }_{1}$} \\
\cline { 1 - 3 } 2 & $b_{2} \cdot e_{1}$ & 2 & \\
\hline 3 & $b_{2} \cdot c_{1}$ & 1 & \\
\hline 4 & $b_{1}$ & 1 & \\
\hline 5 & $c_{1}$ & 1 & \\
\hline 6 & $b_{1} \cdot c_{1} \cdot e_{1}$ & 2 & \multirow{2}{*}{$d_{2}$} \\
\hline 7 & $c_{2}$ & 1 & \multirow{2}{*}{} \\
\hline 8 & $e_{1}$ & 1 & \\
\hline
\end{tabular}

\section{Implementation and experiment}

We implemented the algorithm in Python programming language and tested it using the data in Table 5. The data set has information about stolen cars. The training data has 10 objects $\left\{x_{1}, \ldots, x_{10}\right\}$, three condition attributes \{color, type, origin\}, and a decision attribute \{stolen\}. It is divided into two classes by 'yes' and 'no'. All attributes are categorical.

Table 5. Stolen Car

\begin{tabular}{c|c|c|l|c}
\hline object & color & type & origin & stolen \\
\hline$x_{1}$ & red & sports & domestic & yes \\
\hline$x_{2}$ & red & sports & domestic & no \\
\hline$x_{3}$ & red & sports & domestic & yes \\
\hline$x_{4}$ & yellow & sports & domestic & no \\
\hline$x_{5}$ & yellow & sports & imported & yes \\
\hline$x_{6}$ & yellow & suv & imported & no \\
\hline$x_{7}$ & yellow & suv & imported & yes \\
\hline$x_{8}$ & yellow & suv & domestic & no \\
\hline$x_{9}$ & red & suv & imported & no \\
\hline$x_{10}$ & red & sports & imported & yes \\
\hline & \multicolumn{2}{|c}{}
\end{tabular}

We conducted two experiments. In the first, we compared our algorithm to Naive Bayes classifier by running two algorithms with several different test objects. For example, both algorithms classified $x_{\text {new } 1}=$ \{red, suv, domestic\} to 'no' as shown in Table 6. However, Naive Bayes classifier failed to classify another object $x_{\text {new } 2}=\{$ blue, suv, domestic $\}$ to a class due to the zero probability problem created by the attribute value blue. We need to use some type of data modification, such as Laplace correction [3] to solve this problem. On the other hand, our algorithm successfully classified $x_{n e w 2}$ using the class differentiators listed below.

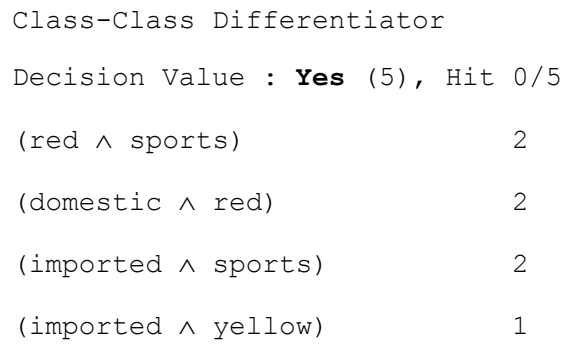


Decision Value : No (5), Hit $3 / 5$

$\begin{array}{ll}\text { (domestic) } & 1 \\ (\text { domestic } \wedge \text { yellow) } & 2 \\ (\text { suv }) & 1 \\ (\text { domestic } \wedge \text { suv) } & 1 \\ (\text { red } \wedge \text { suv) } & 1\end{array}$

In Table 5, the attribute value set $\{$ suv, domestic $\}$ is found only in $x_{8}$, and it works as a class differentiator of $X_{n o}$. This term is a subset of $x_{\text {new2 }}=\{$ blue, suv, domestic $\}$, and is used to predict the decision value of $x_{\text {new2 }}$ to 'no'.

Table 6. Classification of $\mathbf{X}_{\text {new1 }}$ and $\mathbf{X n w}_{\text {new2. }}$

\begin{tabular}{|l|l|l|}
\hline Algorithm & $\boldsymbol{x}_{\text {new1 }}$ & $\boldsymbol{x}_{\text {new }}$ \\
\hline Naive Bayes & yes : 0.037 & yes $: 0.0$ \\
\cline { 2 - 3 } & no $: \mathbf{0 . 0 6 9}$ & no: $: 0.0$ \\
\hline Class Differentiator & yes : 0.4 & yes $: 0.0$ \\
\cline { 2 - 3 } & no $: \mathbf{0 . 8}$ & no $: \mathbf{0 . 6}$ \\
\hline
\end{tabular}

We also compared our algorithm to a rule based classifier. Although the details vary, most rule based classifiers generate a set of if-then rules to predict the unknown data [11]. As described earlier, we often need to run a rule extraction algorithm several times to obtain the rule set that can classify an object. In this experiment, we extracted two sets of rules using ERID [14] and ran Chase [13] algorithm to classify $x_{\text {new } 2}=\{$ blue, suv, domestic $\}$. As shown in Table 7, we could classify $x_{\text {new2 }}$ to $X_{\text {no }}$ using ERID rule set \#1 (that is generated with support $=3$ and confidence $=0.75$ ). However, no decision value was predicted with another rule set (rule set \#2 with support $=2$ and confidence $=0.8$ ) because the terms in rule set \#2 did not have a matching attribute value. The proposed algorithm does not have this problem because the class differentiators are not dependent on input parameters such as threshold values.

Rules generated by ERID

[Rule set \#1] min support 3, confidence 0.75

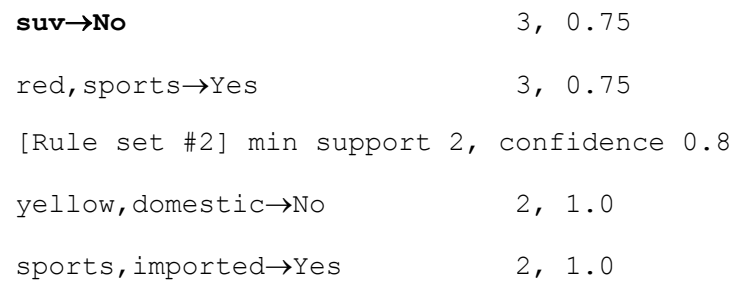

Table 7. Classification of Xnew2 using Chase

\begin{tabular}{|l|c|c|}
\hline & $\sup =3, \operatorname{con} f=0.75$ & $\sup =2$, conf $=0.8$ \\
\hline Rule Based Classification & yes $: 0.0$ & yes $: 0.0$ \\
\cline { 2 - 3 } & no $: \mathbf{1 . 0}$ & no $: 0.0$ \\
\hline
\end{tabular}

\section{Conclusion}

This paper discussed a method for the supervised classification of categorical data. The algorithm uses class differentiators to find out the class label of a test object. The class differentiators are the set of 
attribute values in the training data set that distinguishes one class from the others. The proposed algorithm measures the similarity between the test object and the class differentiators to determine the class membership. The comparison of our algorithm with a rule based classifier and Naive Bayes classifier shows that the proposed algorithm produces more consistent results and less prone to the zero probability problem.

\section{REFERENCES}

[1]. Pawlak, Z., "Rough sets", International Journal of Computing and Information Sciences, 11(5), pp. 341-356, 1982

[2]. Pawlak, Z., Skowron, A., "Rough sets and Boolean reasoning" Information Sciences, 177(1), pp. 41-73, 2007

[3]. Duda, R., Hart, P. and Stork, D., "Pattern classification", Wiley, New York, 2nd edition, 2001

[4]. Tan, P., Steinbach, M. and Kumar, V., "Introduction to data mining", 1st edition, Pearson Addison Wesley, Boston, 2005

[5]. Boriah, S., Ch, ola, V. and Kumar, V., "Similarity measures for categorical data: A comparative evaluation", In: SIAM Data Mining Conference, pp.243-254, 2008

[6]. Dougherty, J., Kohavi, R., Sahami, M., "Supervised and unsupervised discretization of continuous features", In: Twelfth International Conference on Machine Learning. pp.194-202, 1995

[7]. Guarino, N., "Formal ontology, conceptual analysis and knowledge representation", International journal of human-computer studies, 43(5), pp.625-640, 1995

[8]. Xiaodan Zhang, Liping Jing, Xiaohua Hu, Michael Ng, and Xiaohua Zhou. "A comparative study of ontology based term similarity measures on PubMed document clustering", In: 12th international conference on Database systems for advanced applications, pp.115-126, 2007

[9]. Brickell, J. and Shmatikov, V., "The cost of privacy: destruction of data-mining utility in anonymized data publishing" In: ACM SIGKDD international conference, pp.70-78, 2008

[10]. Im, S., "Privacy aware data management and chase", Fundamenta Informaticae, 78(4), pp.507-524, 2007

[11]. Charu C. Aggarwal, and ChengXiang Zhai., "A Survey of Text Classification Algorithms", Mining Text Data, Springer, pp 43-76, 2012

[12]. Posthoff, Ch. and Steinbach, B., "Logic Functions and Equations - Binary Models for Computer Science", Springer, Dordrecht, 2004

[13]. Dardzinska, A. and Ras, Z., "Rule-based Chase algorithm for partially incomplete information systems", In: Second International Workshop on Active Mining, pp.42-51, 2003

[14]. Dardzinska, A. and Ras, Z.W., "Extracting Rules from Incomplete Decision Systems: System ERID" In: Foundations and Novel Approaches in Data Mining, Studies in Computational Intelligence, Vol. 9, Springer, pp.143-154, 2006 